\title{
Development of an electrical exploration data post-processor
}

\author{
Yuri Ignatov ${ }^{*}$, Oleg Tailakov ${ }^{2}$, Evgeniy Saltymakov ${ }^{2}$, Daniil Gorodilov $^{2}$ \\ ${ }^{1}$ T.F. Gorbachev Kuzbass State Technical University. 650000, Russian Federation, Kemerovo, \\ Vesennaya str., 28 \\ ${ }^{2}$ Federal Research Center of Coal and Coal chemistry of Siberian Branch of the Russian Academy of \\ Sciences. 650065, Russian Federation, Kemerovo, Ave. Leningradskiy, 10
}

\begin{abstract}
In modern times, the development of geology and geophysics is associated with complex experiments. The results of these experiments are large arrays of numerical data, which require processing and further analysis. If to process these data manually, it can be a very difficult and routine task. For such studies, specialized tools are important, which are necessary to significantly speed up the processing process and to render visualization of geophysical data in real time. The software is worked out to automate the geophysical data processing obtained after electrical exploration procedure. The designed postprocessor performs functions of data correction and geological and geophysical profile visualization. The user interface of the program provides researchers with the ability to interactively process the initial geophysical data.
\end{abstract}

\section{Introduction}

The general goal of geological exploration is scientifically substantiated, systematic and cost-effective provision of the mining industry with proven reserves of minerals, the study of methods for their complete, comprehensive and economically rational recovery during deposits development with regard of environmental protection. In the process of performing such works, the study of coal seams arrangement, the conditions of their formation and composition [1] is carried out. In addition, the components accompanying the mineral deposits are studied, including rare metals, associated gas, sulfur, etc., the possibility of their extraction or utilization is being considered [2].

The results of such works are geophysical data arrays that require further processing [3]. Manual data processing can be difficult and take inappropriate research time [4]. Using automated processing, the postprocessor performs the functions of interpreting the obtained numerical results [5] and its main tasks are data correction and visualization of the geophysical profile. The postprocessor performed functions speed up the editing of the initial numerical data, and in this approach, researchers can focus only on the exploration works analysis [6]. This work is devoted to the development of software for postprocessing of geophysical data obtained after electrical exploration.

\footnotetext{
*Corresponding author: tailakovov@kuzstu.ru
} 


\section{Materials and Methods}

The initial data obtained from geophysical surveys present an array of values, the analysis of which is possible only during their processing. In Figure 1, the values of this data are presented on visualization, where a visual matrix is drawn depending on the range of colors and values.

The visualization in Figure 1a shows low information content and for greater clarity, it is necessary to interpolate the data. One can compare some interpolation types Linear interpolation is the determination of coefficients of a straight line passing through two specified points [7]. Point values are determined using the straight line formula. With this interpolation, the coefficients are found exclusively vertically or horizontally. The resulting visualization can be seen in Figure 1b.

Bicubic interpolation - in computational mathematics implies the extension of cubic interpolation in case two variables are in function, the values of which are given on a twodimensional regular grid [8]. The surface resulting from bicubic interpolation is a smooth function at the boundaries of neighboring squares (see Figure 1c), in contrast to surfaces resulting from the linear interpolation.

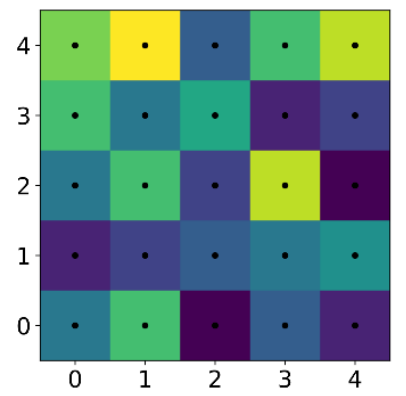

(a)

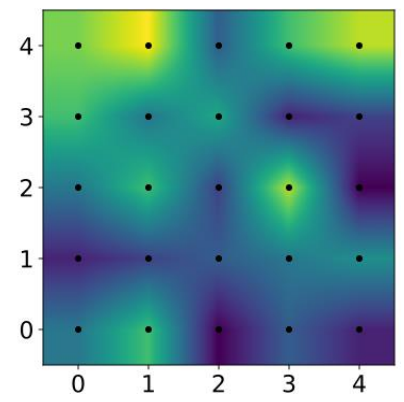

(b)

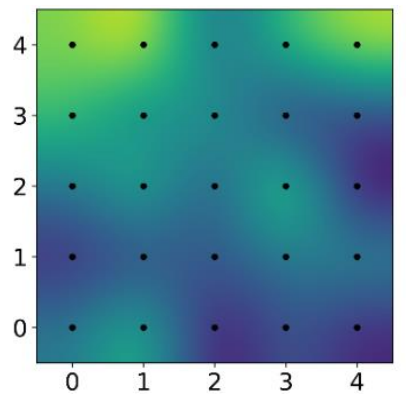

(c)

Fig. 1. Visualization types: a) Visualization of electrical exploration data in a small area; b) Example of linear interpolation; c) Example of bicubic interpolation.

It should be noted that other types of interpolation based on a regular grid can also be used and they improve the visualization clarity, but are not informative enough for data analysis [8].

There is also another way to plot visualization, called triangulation, in which it is necessary to build a surface glued from triangles that connect original points (see Figure 2).
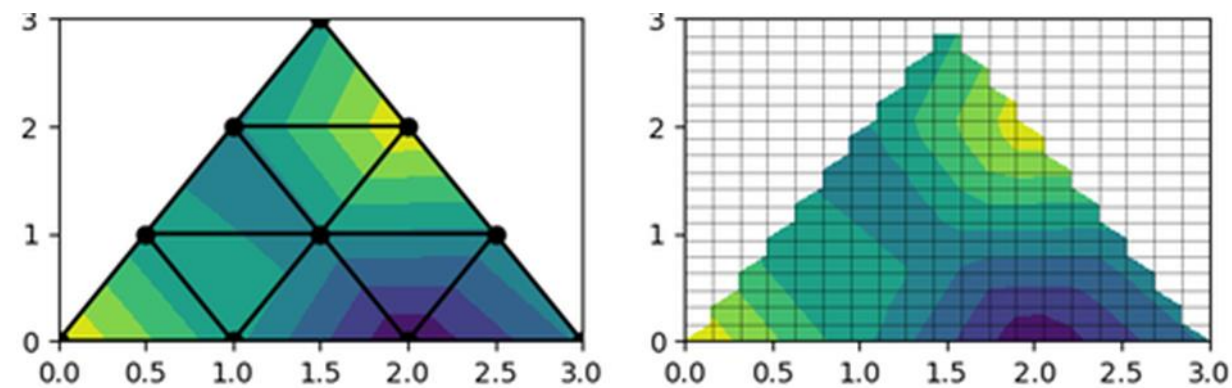

Fig. 2. The visualization of numerical data, triangulation-based interpolation. 
First iteration

\begin{tabular}{|c|c|c|c|c|c|c|}
\hline 2 & -6 & 3 & 1 & 6 & 2 & 3 \\
\hline 0 & 3 & 7 & 8 & 5 & 4 & 0 \\
\hline 0 & 0 & 6 & 4 & 3 & 0 & 0 \\
\hline 0 & 0 & 0 & 5 & 0 & 0 & 0 \\
\hline
\end{tabular}<smiles>O=C1CCCC1</smiles>

\begin{tabular}{|l|l|}
\hline 2 & 6 \\
\hline 1 & 3 \\
\hline
\end{tabular}

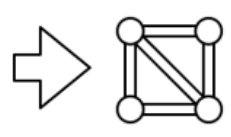

\section{Second iteration}

\begin{tabular}{|l|l|l|l|l|l|l|}
\hline 2 & 6 & -3 & 1 & 6 & 2 & 3 \\
\hline 0 & 3 & 7 & 8 & 5 & 4 & 0 \\
\hline 0 & 0 & 6 & 4 & 3 & 0 & 0 \\
\hline 0 & 0 & 0 & 5 & 0 & 0 & 0 \\
\hline
\end{tabular}

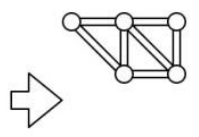

\begin{tabular}{|l|l|}
\hline 0 & 6 \\
\hline 2 & 3 \\
\hline
\end{tabular}

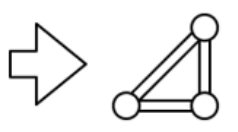

$\vdots$

\section{Last iteration}

\begin{tabular}{|l|l|l|l|l|l|l|}
\hline 2 & 6 & 3 & 1 & 6 & 2 & 3 \\
\hline 0 & 3 & 7 & 8 & 5 & 4 & 0 \\
\hline 0 & 0 & 6 & 4 & 3 & 0 & 0 \\
\hline 0 & 0 & 0 & 5 & 0 & 0 & 0 \\
\hline
\end{tabular}

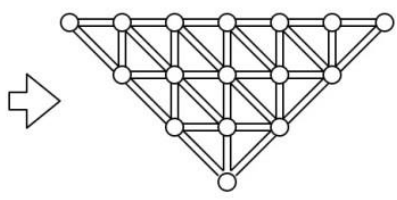

(a)

\begin{tabular}{|l|l|}
\hline 2 & 6 \\
\hline 0 & 3 \\
\hline
\end{tabular}<smiles>C1CCCCC1</smiles>

\begin{tabular}{|l|l|}
\hline 7 & 6 \\
\hline 2 & 0 \\
\hline
\end{tabular}<smiles>O=C1CCCCO1</smiles>

(b)

Fig. 3. Examples of plotting: a) triangulation; b) polygon.

To plot this visualization (see Figure 2), it is necessary to transform the initial geophysical data into a triangulation representation first by applying the construction of a polygonal area.

Iteratively, in two cycles over rows and columns, a grid is built, where adjacent elements are checked for each element of the iteration. The iteration algorithm is clearly shown in Figure 3a. On each selected element, neighboring values are checked, and if these values refer to natural numbers, then based on them, the vertices and edges of the polygon are indicated, and otherwise the construction of the polygon does not occur.

Figure $3 \mathrm{~b}$ demonstrates examples of 4 polygons, depending on the location of the zero element, a certain triangular polygon is built, and if this element is absent, then two triangular polygons are immediately built. Thus, applying the described algorithm and forming a polygonal area, the data required for visualization is processed (see Figure 4).

\begin{tabular}{|l|l|l|l|l|l|l|}
\hline 2 & 6 & 3 & 1 & 6 & 6 & 6 \\
\hline 0 & 3 & 7 & 8 & 5 & 4 & 0 \\
\hline 0 & 0 & 6 & 4 & 3 & 0 & 0 \\
\hline 0 & 0 & 0 & 5 & 0 & 0 & 0 \\
\hline
\end{tabular}
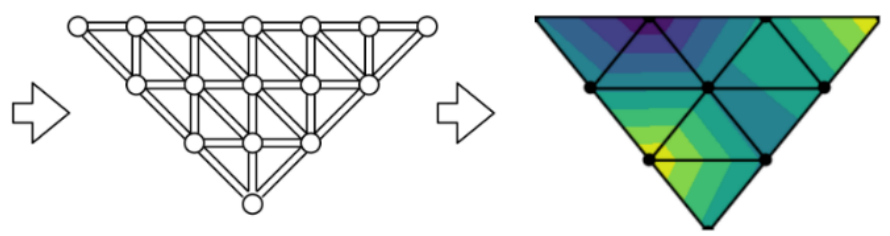

Fig. 4. Visualization plotting stages. 


\section{Results and Discussion}

The software implementation is a desktop application. On the client's personal computer, the user installs the software and launches the desktop application. In this application, the client and server parts are launched.

Figure 5 shows a component diagram, which is similar to a location diagram, where placement objects are detailed with the described components. Main Process of the client application creates web pages by instantiating Browser Window instance. Each Browser Window instance launches the web page in its own Render Proccess. When the Browser Window instance is destroyed, the corresponding rendering process also ends. The main process manages all web pages and related rendering processes. Each rendering process is isolated and responsible only for the web page running in it

The server application implements the computational part for geophysical data processing. For client-server interaction with external clients, the software interface REST API is provided, which uses the HTTP protocol [10].

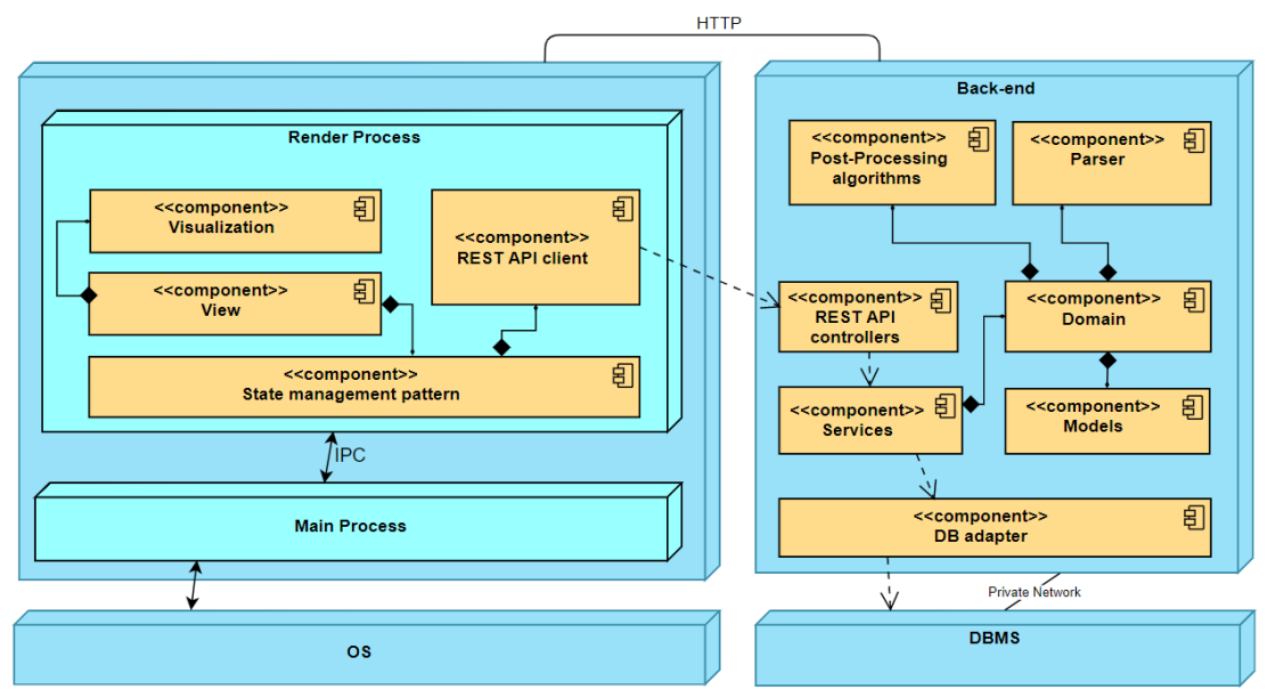

Fig. 5. Component diagram.

Figure 6 demonstrates the software user interface, which has two presentation modes: visualization and editing modes, in which the user has access to tools for interactive viewing and editing of geophysical data.

In the edit mode, the user, by specifying the coordinates of the point being edited, changes the specific resistivity value, and in real time it is possible to view the change introduced into the geophysical profile. 


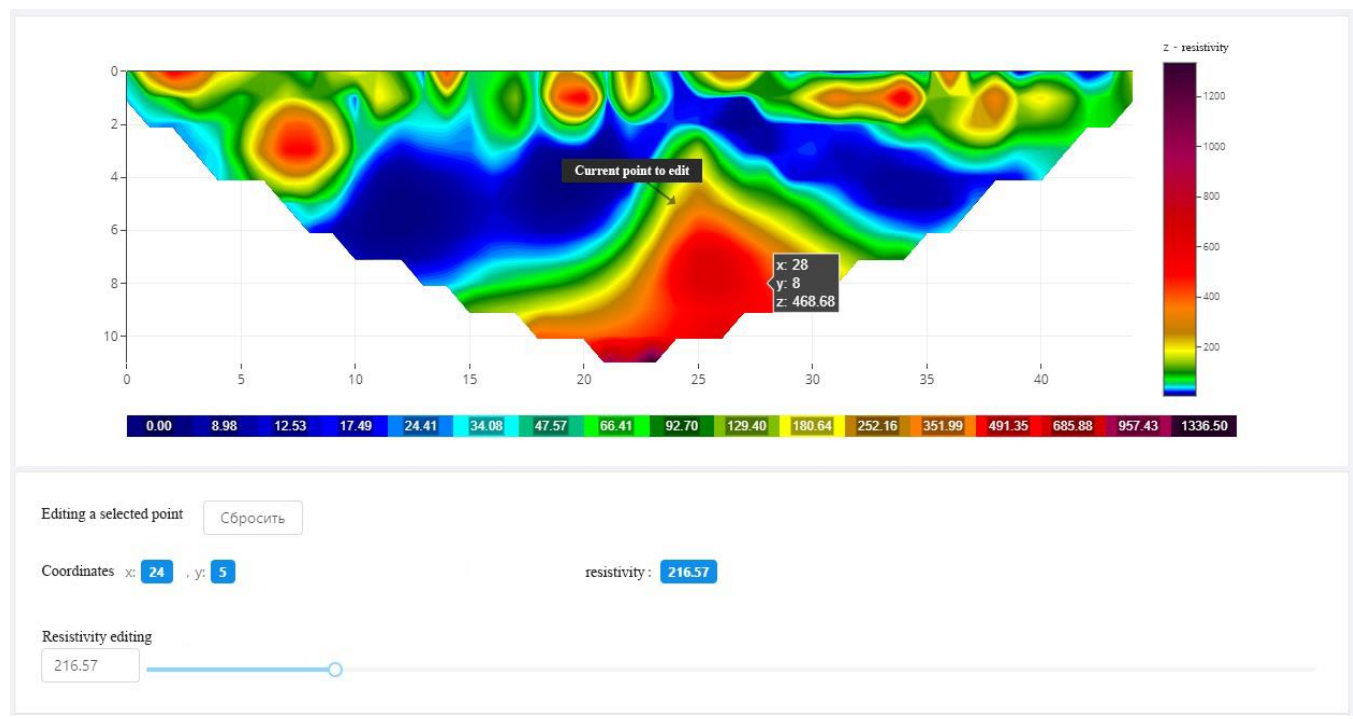

Fig. 6. Editing mode of geological and geophysical section data.

Figure 7 shows visualization of highlighted parts of the geological profile: under $(a, c)$ the initial data are indicated, under $(b, d)$ - the processed data.

a)

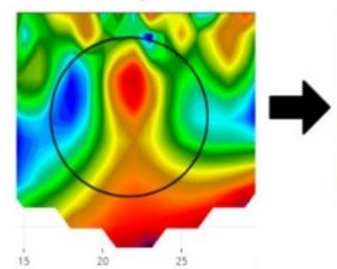

b)

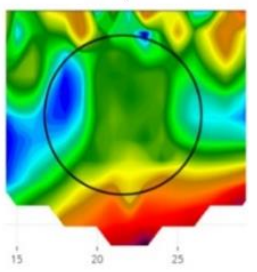

c)

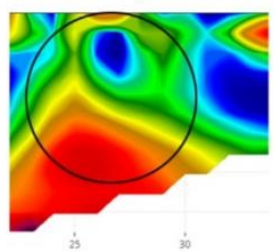

d)

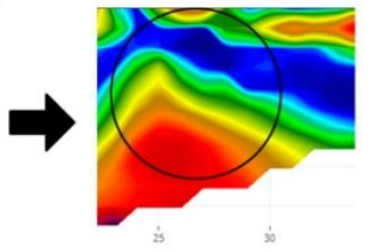

Fig. 7. An example of data processing: (a, c) - initial data, (b, d) - processed data.

\section{Conclusion}

In the course of undertaken work, a study on geological profile visualization was carried out and software developed to automate the processing of geophysical data obtained after electrical exploration procedure. The developed software has accelerated the processing of numerical data and appears as an interactive tool for researchers in geophysical data processing.

\section{References}

1. O.V. Tailakov, D.N. Zastrelov, Ye.A. Saltymakov, M.P. Makeev, S.V. Sokolov, A.S. Yarosh, Aquifers depth detection by electron tomography method in kuzbass conditions, 2 (2016)

2. D.N. Zastrelov, A.N. Kormin, E.A. Saltymakov, O.V. Tailakov, S.V. Sokolov, Determination of geological conditions of gassy coal seams on the basis of seismic acoustic profiling in underground mine workings, 4 (2018)

3. V.L. Averbukh, IEEE Symposium on Human-Centric Computing: Proceedings: IEEE Symposia on Human-Centric Computing Languages and Environments, 390 (2001) 
4. A.V. Azarov, A.V. Patutin, 5th ISRM Young Scholars' Symposium on Rock Mechanics and International Symposium on Rock Engineering for Innovative Future, 944 (2019)

5. V.L. Averbukh, AIP Conference Proceedings: Zababakhin scientific talks - 2005: International Conference on High Energy Density Physics, 481 (2005)

6. L. Zhao, J. Xia, Q. Duan, Advances in Geosciences, 51 (2011)

7. H.I. Alhabbash, A method of characteristics program and post-processor (1994)

8. R. Keys, IEEE Transactions on Acoustics, Speech, and Signal Processing, 1153 (1981)

9. F. Santos, R. Seidel, Journal of Combinatorial Theory, Series A, 186 (2003)

10. M. Heller, Proc. ofthe 4th Intern. Symp. on Spatial Data Handling, 163 (1990)

11. L. Bianchi, S. Turchi, L. Ciofi, IEEE Journal of Biomedical and Health Informatics, 885 (2014) 